\title{
Designing Interference based Routing Metric In Wireless Mesh Network and Comparison According to Various Tunable Parameters
}

\author{
Abhisek Paul ${ }^{1, *}$, Paritosh Bhattacharya ${ }^{1}$ and Santi Prasad Maity ${ }^{2}$ \\ ${ }^{1}$ Department of Computer Science and Engineering, National Institute of Technology, Agartala, India \\ ${ }^{2}$ Department of Information Technology, Bengal Engineering and Science University, Shibpur, India
}

Received: 20 Jul. 2013, Revised: 25 Sep. 2013, Accepted: 27 Sep. 2013

Published online: 1 Jan. 2014

\begin{abstract}
Achieving good performance in wireless mesh network (WMN), implementation of routing metrics is very important. Multiple channels among the multiple radio improve the capacity of the network. Interference based routing metric such as Weighted Cumulative Expected Transmission Time (WCETT), Weighted Cumulative Consecutive Expected Transmission Time (WCCETT), are being proposed in multi-channel multi-hop wireless mesh networks. In this paper we proposed a new interference based routing metric called Modified Weighted Cumulative Consecutive Expected Transmission Time (ModWCCETT) with respect to the selection of less interference path with more channel availability in wireless mesh network. The theoretic analysis and calculations verify the better performance with respect to the other routing metrics.
\end{abstract}

Keywords: Wireless Mesh Network, Multi-Channel, Routing Metric,Multi-Hop,Interference

\section{Introduction}

Wireless Mesh Networks (WMNs)[1]are collection of mesh routers and mesh clients. Mesh routers have minimal mobility and form the backbone of WMNs. Mesh clients can be either motionless or mobile, and can form a client mesh network among themselves and with mesh routers. Wireless mesh networks are basically of three types according to architecture and topology: Client Mesh Networks, Infrastructure Mesh Networks, and Hybrid Mesh Networks [2].

Routing in wireless networks is an active part of research for recent years. Different routing protocols for Mobile Ad-hoc Network [3].

On-demand or reactive routing protocols namely DSR [4], AODV [5] only create a route between a pair of source and destination nodes when the source node really needs to send packets to the destination. Network wide flooding is regularly used to discover routes whey they are needed. For Ad-hoc networks, since there are frequent link breaks caused by the mobility of nodes, flooding-based route discovery provides high network connectivity and comparatively low message overhead compared to proactive routing protocols. However, in mesh networks, links usually have much longer probable lifetimes due to the static nature of mesh routers. On the other hand, in proactive routing protocols, such as OLSR [6], each node maintains tables containing routing information to every other node in the network. All nodes update these tables to maintain a steady and up-to-date view of the network. When the network topology changes, the nodes propagate update messages throughout the network to preserve consistent and up-to-date routing information about the whole network. These routing protocols vary in the method by which packets are forwarded along routes $[1,7,8,9,10,11,12,4$, $5,3,13,6,2,14,15]$.

A good routing metric must accurately capture the quality of network links and aid in computation of good quality paths. Interference is one of the most important characteristics of a routing metric for WMNs.

The reminder of the paper is organized as follows. Section 2 reviews routing metric components in mesh networks. Section 3 describes metric characteristics. Section 4 reviews the existing related works. In Section 5 we propose the routing metric ModWCCETT and

\footnotetext{
*Corresponding author e-mail: abhisekpaul13@gmail.com
} 
compare it with some other existing routing metrics. Section 6 shows simulation and analysis. Section 7 gives conclusion.

\section{Metric Components}

In this section, the key components that can be utilized to compose a routing metric for mesh networks namely Number of hops, Link Capacity, Link Quality and Channel Diversity are discussed.

\subsection{Number of Hops}

Hop count can serve as a routing metric in itself, but can also be a component in a more complex metric. Hop count tends lead to congestion and hot spots $[16,17]$.

\subsection{Link Capacity}

Measuring the link capacity gives a view at the current throughput capability of a link. There are a few ways this can be done. One way is, probe the link actively for measuring transfer speeds and to rely on the current rate of radio interface[18].

\subsection{Link Quality}

High-quality links will form a high-quality path having higher transfer speeds and lower error rates. One way to calculate Link quality is Signal to Noise Ratio (SNR) and Packet Loss Rate (PLR)[7].

\subsection{Channel Diversity}

Use of same channel by consecutive hops of a path may result in significant co-channel interference and reduction of overall throughput. Ideally, all links of a path within interference range of each other should be operating on non-overlapping channels, resulting in significant performance gains. The extent to which this can be achieved can be expressed as channel diversity. Obviously, channel diversity is only relevant for multi-radio networks, since in single-radio networks all interfaces are required to operate on the same channel to guarantee connectivity[8,19,20,21].

\section{Metric Characteristics}

A good routing metric must accurately capture the quality of network links and aid in computation of good quality paths. This section presents desirable characteristics of a good routing metric for WMNs.

\subsection{Inter-Flow Interference}

Inter-flow interference is the interference caused by other flows that are in use on the same channels and are competing for the medium. Such Inter-flow interference can outcome in bandwidth starvation for some nodes since these nodes may always experience busy channels. To avoid such starvation, a routing metric must help routing protocols choose paths that can balance not only the traffic load along the path of a flow, but also reduce the inter-flow interference forced in the entire neighboring area[8, 12,22].

\subsection{Intra-Flow Interference}

Intra-flow interference occurs when the radios of two or more links of a single path or flow function on the same channel. Intra-flow interference can be reduced by growing channel diversity. It is done by selecting non-overlapping channels for adjacent hops of a path. Intra-flow interference is not limited to neighboring links and can be experienced over multiple hops because the interference range of a node is typically bigger than a single hop in wireless mesh network[11,22].

\subsection{External Interference}

External interference occurs when a link experiences interference outside of the control of any node in the network. Here, there are two kinds of external interference: Controlled Interference, where other nodes external to the network use networking technologies that overlap with those used by the network, and Uncontrolled Interference, which is caused by is caused by any other source of radio signals emitted in the same frequency range, but not participating in the same MAC protocol. Both of these types of external interference can influence link quality and can seriously affect network performance and throughput $[13,22]$.

\subsection{Locality of Information}

Some metrics require information such as channels used on previous hops of a path, or other metrics observed on other nodes of the networks, such as packet delivery rate or noise levels. This non-local information can be part of routing metric and can be used to make more effective routing decisions. If the metric requires a lot of information from nodes external to itself, the result can be the following two problems. The first one is growing the routing overhead in the network and the Second one is bigger route establishment delays. 


\subsection{Agility}

The agility of a metric refers to its ability to react quickly and efficiently to changes in the network in terms of topology or load. In order for a metric to be considered agile, the rate at which measurements are taken should be higher than the rate of change in the network.

\subsection{Stability}

The metric of a link should not vary too greatly over time. Abrupt changes in the metric can activate a protocol to send out route updates and may cause the protocols overhead to become extremely high. The topology-dependent metrics should be steady, especially in case of low-mobility scenarios $[8,23]$.

\subsection{Throughput}

In general, a metric should be able to select routes with greater throughput consistently. Higher the throughput better will be the performance $[18,17]$.

\section{Related Work}

In this section, we will discuss some of the most important routing metrics in wireless mesh networks.

\subsection{ETX}

The Expected transmission count (ETX) [7]is the estimated number of transmissions (including re transmission) required to send a data packet over the link. The total ETX of a path is summation of ETX of all links on the path. The derivation of ETX starts with measurements of the underlying packet loss probability in both the forward and reverse directions.

Let us consider a wireless link between two nodes A and B. Both nodes are sending a probe [2] packet every second. Let node $\mathrm{A}$ has received nine packets from node $\mathrm{B}$ and node $\mathrm{B}$ received seven packets from node $\mathrm{A}$ in 10sec. After 10sec. node A received an acknowledgement packet containing the number of probes $\mathrm{B}$ has received from A. Based on these count values, node A calculate forward and backward link loss rate as $(10-9) / 10$ and $(10-7) / 9$ respectively. So, the value of ETX is 1.67 in the given link [2].

\subsection{ETT}

The Expected transmission time (ETT) [8,9] is an improvement over ETX. It also considers bandwidth while assigning metric to a link. If $\mathrm{S}$ is the size of the packet and B is bandwidth of the link then.

$$
E T T=E T X \times \frac{S}{B} .
$$

In this way, ETT of the link captures the time taken for successfully transmitting a packet on the link.

\subsection{WCETT}

The Weighed Cumulative Expected Transmission Time (WCETT)[10] metric has been designed to improve the ETT metric by considering channel diversity. The WCETT metric of a path $\mathrm{p}$ is defined as follows:

$$
X_{j}=\sum_{\text {Hop } i \text { is on channel } j} E T T_{i} \quad 1 \leq j \leq k
$$

$$
{ }_{W C E T T}=(1-\beta) \times \sum_{i=1}^{n} E T T_{i}+\beta \times \max _{1 \leq j \leq k} X_{j}
$$

$X_{j}$ is the sum of the ETT values of links that are on channel $j$ in a system. The system has $k$ orthogonal channels. Here $\beta$ is a tunable parameter within the bounds $0 \leq \beta \leq 1$ that controls preference of channel diversity over path length.

\subsection{WCCETT}

The Weighted Cumulative Consecutive Expected Transmission Time (WCCETT) [10]proposes a way to extend WCETT for better consideration of intra-flow interference, with the definition as follows:

$$
\begin{gathered}
Y_{j}=\sum_{\text {Hop } i \text { is on Segment } j} E_{i} T_{i} \quad 1 \leq j \leq k \\
\text { WCCETT }_{P}=(1-\beta) \times \sum_{i=1}^{n} E T T_{i}+\beta \times \max _{1 \leq j \leq k} Y_{j}
\end{gathered}
$$

Like WCETT in this matrix there are two parts. The first part is summation of ETT in the links of a path $P$ and the second part is the channel diversity. $\beta$ is a tunable parameter.

This matrix gives better result than WCETT matrix. The first part is multiplied by $(1-\beta)$ and the second part is multiplied by $\beta$. The value of $n$ is the number of links on path $p . k$ is the number of orthogonal channels. The term Segment is newly included in the metric and only consecutive channels are incorporated in calculation for better result in comparison with other relevant metrics.

A segment is defined as consecutive hops on the same channel. Different segments do not interfere with each other. Thus, WCCETT selects a path with more channel 


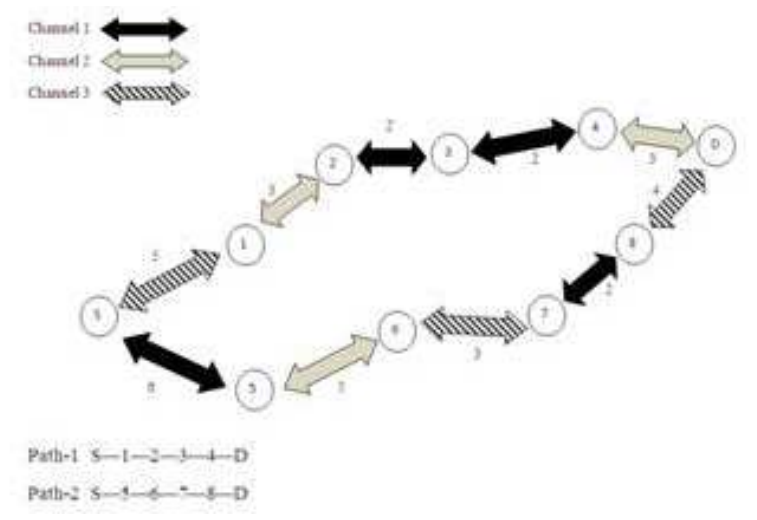

Fig. 1: Multi-Channel Multi- Hop paths between nodes S and D and their ETT values.

diversity (smaller segments) compared to WCETT yielding lesser intra-flow interference. $Y_{j}$ is the sum of the ETT values of links that are on segment $j$ in a system .Where there are $n$ hops with $k$ channels, and $\beta$ is a tunable parameter where $0 \leq \beta \leq 1$.

\section{Proposed Routing Metric}

The modified Weighted Cumulative Consecutive Expected Transmission Time, proposes a way for better consideration of intra-flow interference, ModWCCETT can be expressed as:

$$
\begin{gathered}
Z_{j}=\sum_{\text {Conflicting link is on Channel } j} E T T_{i} \quad 1 \leq j \leq k \\
\text { ModWCCETT } T_{P}=(1-\beta) \times \sum_{i=1}^{n} E T T_{i}+\beta \times \max _{1 \leq j \leq k} Z_{j}
\end{gathered}
$$

A segment in ModWCCETT is defined as conflicting hops on the same channel which interfere with each other. In Figure 1, path 2 has one segment including hop $\{3,4\}$ while path 3 has no segments. Different segments do not interfere with each other. This way, ModWCCETT selects a path with more channel diversity (smaller segments) compared to WCCETT [10] yielding lesser intra-flow interference. $Z_{j}$ is the sum of the ETT values of links that are on segment $j$ in a system. Where there are $n$ hops with $k$ channels, and $\beta$ is a tunable parameter subject to $0 \leq \beta \leq 1$.

Equation 1 represent the expected transmission time of a path with minimum interference. Figure 1 shows multi-channel routing topology with three paths acquiring three channels. Here we find the values of ETT for every link. Figure 2 depicts the comparison of ModWCCETT with respect to various tuning parameters. In Figure 3 and in Figure 4 we find comparison between various routing metrics and these are taken from the routing topology

\begin{tabular}{|c|c|c|}
\hline Mletric & Path & Calculation \\
\hline & 1 & $(0.5) \times 14-(0.5) \cdot \max \{(5),(3-3),(2-1)]=10$ \\
\hline WCETT & 2 & $(0.5) \times 20-(0.5) \times \max ((6-2),(5),(3-4)\}=14$ \\
\hline & 1 & $(0.5) \times 14-(0.5) \times \max ((5),(3))(2-1),(3),=95$ \\
\hline WCCETT & 2 & $(0.5) \times 20-(0.5) \times \max \{(0),(5),(3),(2),(4)\}=13$ \\
\hline & $T$ & $(0.5) \times 14-(0.5) \times \max \{(2-1))=8.5$ \\
\hline ModWCCETI & 2 & $(0.5) \times 20+(0.5) \times 0=10$, no ccallicting link) (max is 0$)$ \\
\hline
\end{tabular}
which is in Figure 1.
Fig. 2: Estimated value of ModWCCETT in various tunaing parameters for three paths.

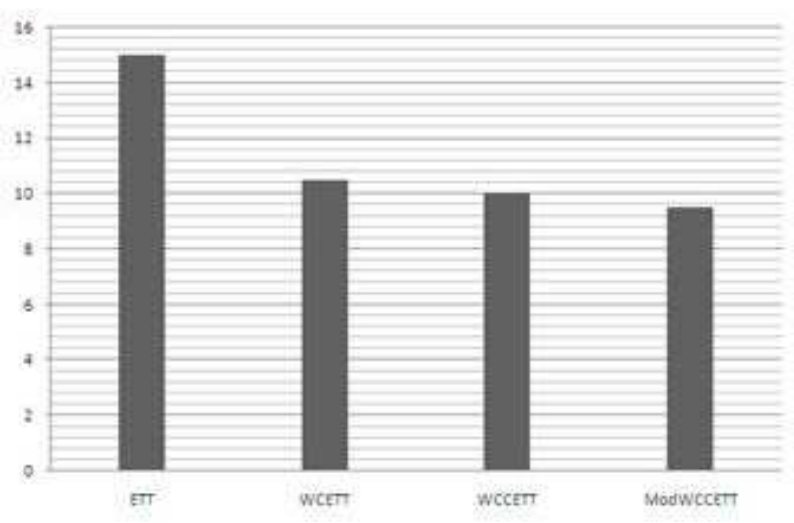

Fig. 3: Comparison of ETT, WCETT, WCCETT and ModWCCETT for Path-1 in the given network topology.

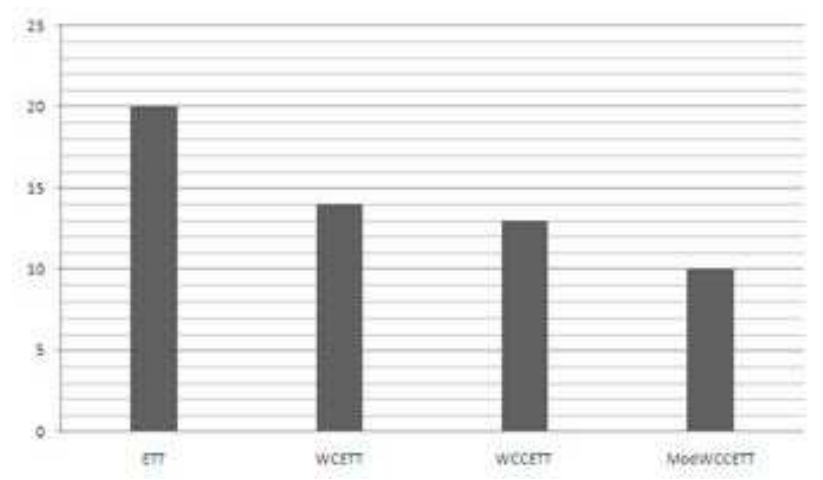

Fig. 4: Comparison of ETT, WCETT, WCCETT and ModWCCETT for Path-2 in the given network topology. 


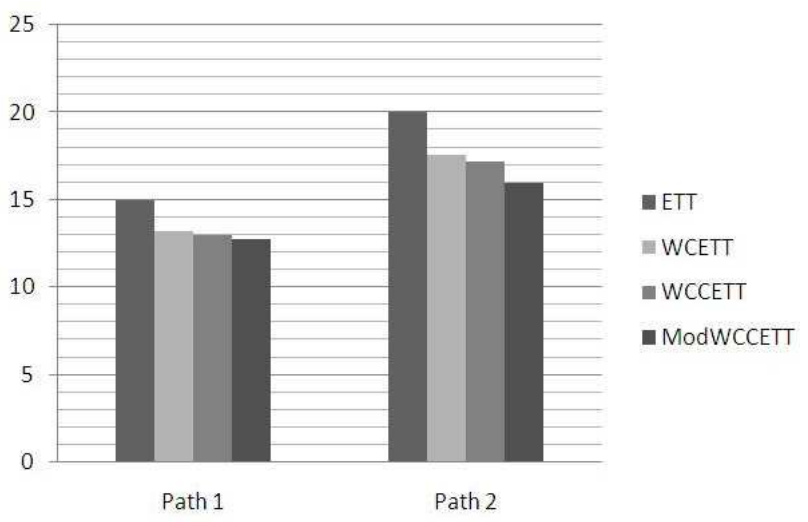

Fig. 5: Comparison of WCETT, WCCETT and ModWCCETT metrics where tunable parameter is 0.2

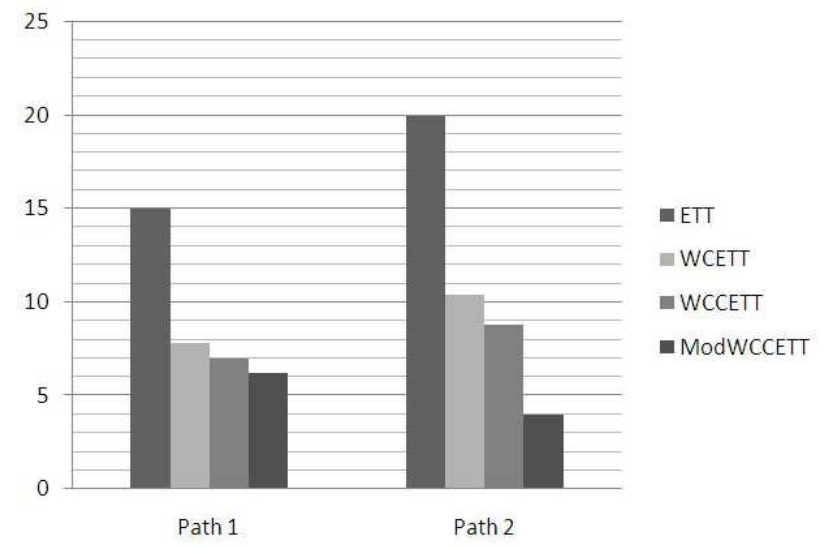

Fig. 6: Comparison of WCETT, WCCETT and ModWCCETT metrics where tunable parameter is 0.8

\section{Comparison and Analysis}

This section describes the performance of ModWCCETT with compared to WCETT and WCCETT. Tunable parameter, $\beta$ is introduced for the comparisons. Range of this parameter is between 0 and 1 . Various tunable parameters are introduced. Here we compared the metrics with tunable parameter 0.2 and 0.8 respectively[24].

Routing in multi-channel and multi-hop wireless mesh network suffer from interference problem. The calculations give some fundamental comparisons between various routing metrics with respect to co-channel interference.

Figure 5 shows that path 1 would be selected for routing because the expected transmission time is less in this path with respect to WCETT, WCCETT, ModWCCETT metrics. Here, Tunable parameter is 0.2 for the routing calculation.
Figure 6 also describe the comparisons of path metrics. Here path 2 would be selected for routing because the transmission time is less in this path with respect to WCETT, WCCETT, and ModWCCETT metrics. Tunable parameter is set 0.5 for the routing calculation.

So, we observed that path selection with less interference through ModWCCETT metric is possible. In Figure 5 and in Figure 6 we find calculation of network with 3-channel and 5-hop. In every analysis the performance of proposed metric is better than other metrics. Metric ModWCCETT has low routing overhead. Intra-flow interference is low in ModWCCET comparing to ETT, WCETT, and WCCETT. Here, we have also seen the value of ModWCCETT metric is better than ETT, WCETT, and WCCETT metrics.

\section{Conclusion}

This paper presents various routing metrics in Multi Channel Multi Hop Wireless Mesh Network. These metrics give end to end transmission time calculation through various equations. Sending data packets to its desired destination we have to choose a route in consideration with minimum end to end delay. We applied mathematical equation for the routing metrics which is called Modified Weighted Cumulative Consecutive Expected Transmission Time (ModWCCETT). We also applied various values as tunable parameters in the calculations for comparison of metrics. Calculation and Analysis show the path selection with respect to interference, the ModWCCETT metric is better than other metrics.

\section{Acknowledgement}

The authors are grateful to the anonymous referee for a careful checking of the details and for helpful comments that improved this paper.

\section{References}

[1] I. F. Akyildiz, X. Wang, and W. Wang, "Wireless mesh networks: a survey", Comput. Netw. ISDN Syst., 47, 445-487 (2005).

[2] Yan Zhang , Jijun Luo ,Honglin Hu,"Wireless Mesh Networking, Architectures, Protocols and Standards", Auerbach Publication, (2007).

[3] S. Bellofiore, J. Foutz, R. Govindarajula, et al., "Smart Antenna System Analysis, Integration and Performance for Mobile Ad-Hoc Networks (MANETs)",IEEE Trans. Antennas Propagat., 50, 571-581 (2002).

[4] D. Johnson, D. Maltz, and J. Jetcheva, "The dynamic source routing protocol for mobile ad hoc networks", Internet Draft,draft-ietf-manet-dsr-07.txt, (2002). 
[5] C. Perkins, E. Belding-Royer, and S. Das, "Ad hoc OnDemand Distance Vector (AODV) Routing”, RFC 3561, (2003).

[6] T. Clausen and P. Jacquet, "Optimized Link State Routing Protocol (OLSR)", RFC 3626, (2003).

[7] D. S. J. D. Couto, D. Aguayo, J. Bicket, and R. Morris, "A high-throughput path metric for multi-hop wireless routing,"in MobiCom 2003: Proceedings of the 9th annual international conference on Mobile computing and networking. New York, NY, USA: ACM, 134-146 (2003).

[8] R. Draves, J. Padhye, and B. Zill, "Routing in multiradio, multi-hop wireless mesh networks",in MobiCom 2004: Proceedings of the 10th annual international conference on Mobile computing and networking. New York, NY, USA: ACM, 114-128 (2004).

[9] J. P. Richard Draves, Brian Zill, "Comparison of Routing Metrics for Static Multi-Hop Wireless Networks", ACM SIGCOMM, 133-144 (2004).

[10] W. Jiang, Z. Zhang, X. Zhong, "High Throughput Routing in Large-Scale Multi-Radio Wireless Mesh Networks", Wireless Communications and Networking Conference, 3598-3602 (2007).

[11] Y. Yang, J. Wang, and R. Kravets, "Designing Routing Metrics for Mesh Networks", IEEE Workshop on Wireless Mesh Networks (WiMesh), Proceedings of the, (2005).

[12] J. Li, C. Blake, S. J. De Couto, H. I. Lee, and R. Morris, "Capacity of AdHoc wireless networks", in Proceedings of the 7th annual international conference on Mobile computing and networking, 61-69 (2001).

[13] R. Kumar and P. Gupta, "The Capacity of Wireless Networks," IEEE Transactions on Information Theory, 46, 388-404 (2000).

[14] Ramanujan K Sheshadri and Dimitrios Koutsonikolas, "Comparison of Routing Metrics in 802.11n Wireless Mesh Networks", 2013 Proceedings IEEE INFOCOM, 1869-1877 (2013).

[15] Stephen Atambire Nsoh and Robert Benkoczi, "Routing and Link Scheduling with QoS in IEEE 802.16 Mesh Networks", IEEE Wireless Communicationsand Networking Conference, (WCNC) NETWORKS, 233-238 (2013).

[16] H. Hassanein and A. Zhou, "Routing with Load Balancing in Wireless Ad hoc Networks", in Proceedings of the 4th ACM international workshop on Modeling, analysis and simulation of wireless and mobile systems Rome, 89-96 (2001).

[17] Yu-Shan Liang, Wei-Ho Chung, Hongke Zhang and Sy-Yen Kuo, "Throughput Improvement of Multi-hop Wireless Mesh Networks with Cooperative Opportunistic Routing?,esignTool", IEEEWireless Communications and Networking Conference (WCNC), 3035-3039 (2012).

[18] B. Awerbuch, D. Holmer, and H. Rubens, "The Medium Time Metric: High Throughput Route Selection in Multi-rate Ad Hoc Wireless Mesh Networks", Mobile Networks and Applications, 11, 253-266 (2006).

[19] P. Bahl, A. Adya, J. Padhye, and A. Wolman, "Reconsidering Wireless Systems with Multiple Radios", SIGCOMM Computer Communication Review, 34, 39-46 (2004).

[20] Jiansheng Qian, Hu Zhong, Xiaolong Feng, Qianqian Hu, "Study on the Routing Metric of Multi-channel Wireless Mesh Network", International Conference on Industrial Control and Electronics Engineering, 1605-1607 (2012).
[21] Xiaoheng Deng,Qiang Liu, Xu Li, Lin Cai and Zhigang Chen, "Channel Quality and Load Aware Routing in Wireless Mesh Network",IEEEWireless Communicationsand Networking Conference (WCNC),NETWORKS, 2068-2073 (2013).

[22] A. P. Subramanian, M. M. Buddhikot, and S. Miller, "Interference Aware Routing in Multi-Radio Wireless Mesh Networks?, ", in Proceedings ofthe 6th ACM international symposium on Mobile ad hoc networking and computing, 6877, (2005).

[23] G. Holland, N. Vaidya, and P. Bahl. A rate-adaptive MAC protocol for multi-hop wireless networks. Mobile Computing and Networking, 236-251 (2001).

[24] A. McAuley, K. Sinkar, L, Kant, C. Graff, M. Patel,’Tuning of Reinforcement Learning Parameters applied to OLSR using a Cognitive Network Design Tool",IEEE Wireless Communications and Networking Conference (WCNC), 2086-2091 (2012). 


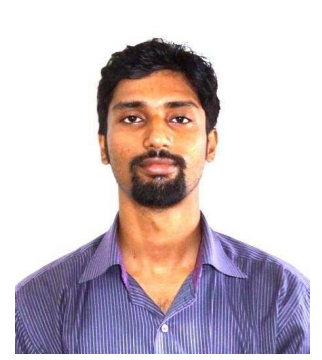

Abhisek Paul received the M.Tech. degree in Computer Science and Engineering at Tripura University of Agartala, India. $\mathrm{He}$ is the $\mathrm{PhD}$ Scholar in the Department of Computer Science and Engineering in National Institute of Technolohy, Agartala, India. His research interests are in the areas of wireless mesh network, mobile adhoc network, vehicular adhoc network, mathematical modeling, applied mathematics including the mathematical methods, numerical methods.

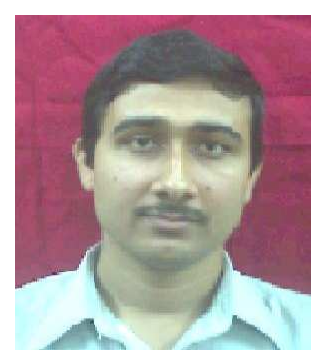

Paritosh Bhattacharya received the $\mathrm{PhD}$ degree in Mathematics for Engineering Science at Jadavpur University of Kolkata, India. $\mathrm{He}$ is the Associate Professor of Department of Mathematics and Head of the Department of Computer Science and Engineering in National Institute of Technolohy, Agartala, India. His research interests are in the areas of wave equation, mathematical modeling, applied mathematics including the mathematical methods and models for complex systems, numerical methods. He has published research articles in reputed international journals of mathematical and engineering sciences. He is referee and editor of mathematical and engineering journals.

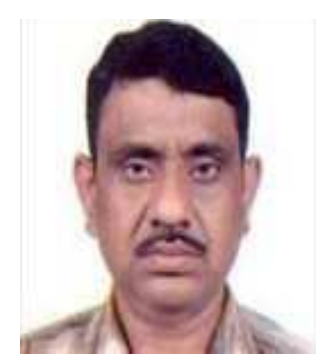

Santi Prasad Maity received the Post-Doctoral degree in Laboratoire des Signaux et Systems at France. $\mathrm{He}$ received the $\mathrm{PhD}$ degree in Engineering (Computer Science and Technology) at BESU, Shibpur, India Bengal Engineering and Science University of Shibpur, India. His research interests are in the areas of digital image watermarking,signal processing, wavelet theory based analysis, soft-computing, access control, reversible watermarking, VLSI Architecture, cognitive radio, spectral sensing and compressive sampling, integration of cognitive radio with wireless network, wireless network, physical layer security of network, power control in information theoretic security,optical information processing, combinational and sequential circuits, codes generation for optical communication. He has published research articles in reputed international journals of engineering science. He is referee and editor of engineering journals. 